\title{
Efficacy of Antibiotic Prophylaxis after Endobronchial Ultrasound-guided Transbronchial Needle Aspiration: A Preliminary Prospective Study
}

Haruhi Takagi ${ }^{1}$, Tetsutaro Nagaoka ${ }^{1 *}$, Katsutoshi Ando ${ }^{1}$, Takeo Tsutsumi ${ }^{1}$, Masako Ichikawa ${ }^{1}$, Ryo Koyama ${ }^{1}, N^{2}{ }^{1}$ Ko Shimada ${ }^{1}$, Kazunori Tobino $^{2}$ and Kazuhisa Takahashi ${ }^{1}$

${ }^{1}$ Department of Respiratory Medicine, Juntendo University Graduate School of Medicine, Tokyo, Japan

${ }^{2}$ Department of Respiratory Medicine, lizuka Hospital, Fukuoka, Japan

\section{Abstract}

Background: Endobronchial ultrasound-guided transbronchial needle aspiration (EBUS-TBNA) is useful for diagnosing mediastinal and hilar lymphadenopathy. While bacteremia and serious infectious complications after EBUS-TBNA have been reported, the efficacy of antibiotic prophylaxis is unclear. This preliminary prospective study aimed to evaluate the efficacy of antibiotic prophylaxis after EBUS-TBNA.

Methods: Patients were randomly assigned to the antibiotic group $(n=43)$ or control group $(n=47)$. Ampicillin/ sulbactam was intravenously administered before the puncture, followed by oral amoxicillin/clavulanate. The primary outcome was body temperature (BT) within 5 days after EBUS-TBNA. The secondary outcomes were the change in the white blood cell count (WBC) and C-reactive protein (CRP) value before and after the examination, and the frequency of infectious complications that required antibiotics. We also assessed the clinical characteristics of patients with fever.

Results: BT after EBUS-TBNA did not significantly differ between the groups. Antibiotic prophylaxis had no effect on the changes in the WBC and CRP value. Neither group had infectious complications associated with EBUS-TBNA. The smoking pack-year was higher in the group with fever than without fever, although the WBC and CRP value at baseline were similar between the groups. The number of punctures, presence of low-density areas in the lymph nodes, and antibiotic treatment were unrelated to the development of fever following EBUS-TBNA.

Conclusion: Results of this study and the low frequency of infectious complications, which has been demonstrated in previous reports, suggest that antibiotic prophylaxis is not recommended for EBUS-TBNA (UMIN: 000008813).

Keywords: Antibiotics; Endobronchial ultrasound-guided transbronchial needle aspiration; Prophylaxis; Postbronchoscopy fever; Infectious complication

\section{Introduction}

Endobronchial ultrasound-guided transbronchial needle aspiration (EBUS-TBNA) is a useful modality in the pathological diagnosis of hilar and mediastinal lymphadenopathy caused by lung cancer [1-3], sarcoidosis [4], malignant lymphoma [5,6], and other diseases $[7,8]$. Recent reports indicate that the diagnostic sensitivity and accuracy of mediastinal lymph nodes in the staging of lung cancer by EBUS-TBNA were similar to the sensitivity and accuracy of mediastinoscopy, and the number of complications was fewer with EBUS-TBNA than with mediastinoscopy [9]. Another group of investigators showed that the diagnostic sensitivity of EBUS-TBNA for metastatic lymphadenopathy of lung cancer was higher than the sensitivity of positron emission tomography [10]. Moreover, the quality of samples from lymph nodes (LNs) obtained by EBUS-TBNA was sufficient for molecular testing, which included epidermal growth factor receptor mutation and the anaplastic lymphoma kinase fusion gene in lung cancer [11-13].

Although EBUS-TBNA is a relatively tolerable and safe procedure [14], infectious complications after EBUS-TBNA have been reported including mediastinitis, pneumonia, and pericarditis [15]. Infectious complications induced by EBUS-TBNA are often more serious than infections associated with conventional fiberoptic bronchoscopy, and precautions should be considered to prevent these infectious events. Whereas prior studies have not indicated the usefulness of antibiotics prophylaxis after conventional fiberoptic bronchoscopy, the efficacy of antibiotic prophylaxis especially after EBUS-TBNA remains unclear. Thus, the objective of this study was to evaluate the effect of antibiotic prophylaxis in preventing the frequency of postbronchoscopy fever and infectious complications after EBUS-TBNA, preliminarily.

\section{Materials and Methods}

\section{Study design}

This open-label, prospective, randomized controlled trial was conducted from January 2011 to December 2013 at the Department of Respiratory Medicine of Juntendo University Hospital (Tokyo, Japan) and at Iizuka Hospital (Fukuoka, Japan). The study protocol was reviewed by the independent ethics committee of the institutional review board at each center. All patients provided written informed consent. The trial was registered with UMIN (000008813).

\section{Patients}

The eligibility criteria for participation were an age of 20 years or older, adequate liver and renal function, and written informed consent to participate. The key exclusion criteria were a history of allergy to penicillin, suspected active bacterial infection, a body temperature (BT) above $37.8^{\circ} \mathrm{C}$, and use of immunosuppressive or anticarcinogenic agents or non-steroidal anti-inflammatory drugs. Patients who had significant hematological, hepatic or renal abnormalities or who were pregnant or breast feeding were also excluded from the study. The

*Corresponding author: Tetsutaro Nagaoka, Department of Respiratory Medicine, Juntendo University Graduate School of Medicine 2-1-1 Hongo Bunkyoku, Tokyo-113-8421, Japan, Tel: +81-3-5802-1063; E-mail: jnagaoka@juntendo.ac.jp

Received July 17, 2017; Accepted July 19, 2017; Published July 24, 2017

Citation: Takagi H, Nagaoka T, Ando K, Tsutsumi T, Ichikawa M, et al. (2017) Efficacy of Antibiotic Prophylaxis after Endobronchial Ultrasound-guided Transbronchial Needle Aspiration: A Preliminary Prospective Study. J Pulm Respir Med 7: 416. doi: 10.4172/2161-105X.1000416

Copyright: $\odot 2017$ Takagi $\mathrm{H}$, et al. This is an open-access article distributed under the terms of the Creative Commons Attribution License, which permits unrestricted use, distribution, and reproduction in any medium, provided the original author and source are credited. 
patients' comorbidities were assessed using the Charlson Comorbidity Index (CCI) [16].

\section{Endobronchial ultrasound-guided transbronchial needle aspiration and antibiotic treatment}

No patient was intubated with an endobronchial tube for EBUSTBNA. After conventional fiberoptic bronchoscopy for intrabronchial anesthesia and observation, EBUS-TBNA was performed using a convex probe endobronchial ultrasound bronchoscope (BF-UC260FW; Olympus, Tokyo, Japan). A dedicated 21- or 22-gauge needle (NA201SX-4021 and NA-201SX-4022, respectively; Olympus, Tokyo, Japan) were used for the punctures. The stations of the punctured LNs were $\# 2 \mathrm{R}, \# 4 \mathrm{R}, \# 4 \mathrm{~L}, \# 7$, and bilateral \#10 and \#11. All procedures were performed as previously described [17].

In the antibiotics group, ampicillin/sulbactam $(3 \mathrm{~g}$, administered once) was intravenously administered $30 \mathrm{~min}$ before the puncture, followed by oral amoxicillin/clavulanate $(250 \mathrm{mg}$, three times daily for 7 days). No antibiotic was administered to the control group. We selected the aforementioned antibiotics for prophylaxis because pathogenic bacteria in infectious complications associated with EBUSTBNA were mostly caused by normal oral flora organisms, and the spectra of the selected antibiotics are sufficient for these bacteria.

\section{Outcome measurements}

The enrolled patients measured and recorded their own axillary BT after $12 \mathrm{~h}$, and then 2 days, 3 days, 4 days, and 5 days after the procedure. We defined "fever" as a BT above $37.8^{\circ} \mathrm{C}$, based on the definition in a previous textbook [18]. The primary outcome of this study was the inhibitory effects of antibiotic prophylaxis on postbronchoscopy fever after EBUS-TBNA. The secondary outcomes included the change in the white blood cell count (WBC) and C-reactive protein (CRP) value between the baseline and after examination within 14 days and the frequency of pulmonary or mediastinal infections that required antibiotic treatment within 28 days. We also assessed the clinical characteristics of patients without fever or with fever after EBUS-TBNA. Risk factors of postbronchoscopy fever were evaluated by univariate analysis between the groups without and with fever with regard to smoking history, presence of comorbidities and antibiotic prophylaxis, the baseline WBC and CRP value, the number of punctures, and the presence of low-density areas in the LNs on computed tomography.

\section{Statistical analyses}

Sample size was calculated based on the primary outcome by Fisher Exact Test. After the conventional fiberoptic bronchoscopy, incidence of respiratory tract infection following the prophylactic antibiotics treatment was $0.7 \%$ [19], and frequency of postbronchoscopy fever were $10.5-26.6 \%$ in past decade reports $[20,21]$. Based on these data, 102 patients ( $n=51$ in each group) was required at $80 \%$ power and $\alpha=0.05$ to determine the preventive effect of postbronchoscopy fever by antibiotic prophylaxis.

We used the chi-square test or the Mann-Whitney $U$ test, as appropriate, to compare the patients' characteristics. We analyzed the serial changes in the BT by using linear mixed models. Serial changes in the WBC and CRP values were compared by using the Wilcoxon signed-rank test. These analyses were performed using Statistical Package for Social Science (SPSS) Version 21 software (Chicago, IL). A $\mathrm{p}$ value less than 0.05 was considered significant.

\section{Results}

\section{Study profile and baseline characteristics}

One hundred fourteen patients were eligible for this study. 60 patients were assigned to the prophylactic antibiotic group and 54 patients were assigned to the control group (i.e., no antibiotics). Eleven patients in the control group did not proceed because of withdrawals or protocol violations, and 13 patients in the antibiotics group were excluded because of protocol violations after registration. In the end, 47 patients in the control group and 43 patients in the antibiotics group participated in this study (Figure 1). The baseline characteristics of both groups are shown in Table 1. Age, sex, and smoking history were similar in both groups. The CCI value, BT, WBC, and CRP value at baseline did not significantly differ between the groups.

\section{The effect of antibiotic prophylaxis on post-EBUS-TBNA fever}

The BT values after EBUS-TBNA are shown in Figure 2. Patients with a fever at baseline were excluded. Thus, we compared the BT after EBUS-TBNA between the group with and the group without prophylactic antibiotics. Body temperature was highest at 12 hours after

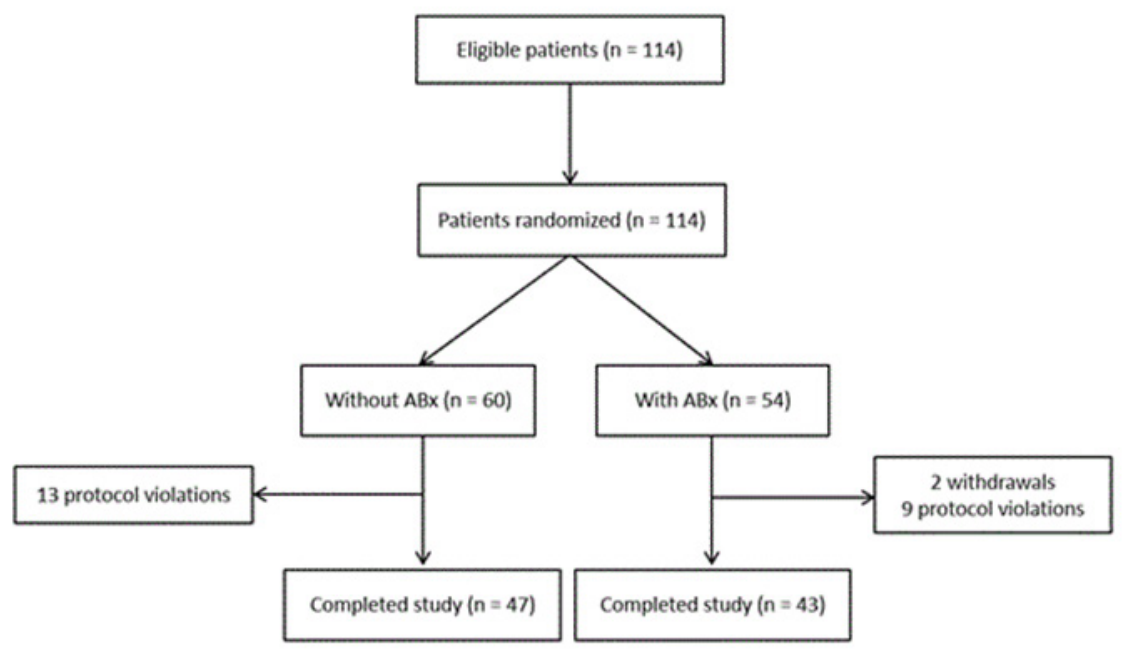

Figure 1: Study profile-The flow chart shows the randomization process for the 114 eligible patients in this study. 


\begin{tabular}{|c|c|c|c|}
\hline & Without Abx $(n=47)$ & With Abx (n=43) & $p$ value \\
\hline Age $(y)$ & $59.3 \pm 13.8$ & $58.7 \pm 15.3$ & 0.9 \\
\hline Male sex & $28(60)$ & $29(67)$ & 0.513 \\
\hline \multicolumn{4}{|l|}{ Smoking history } \\
\hline Never smoker & $7(15)$ & $5(12)$ & 0.761 \\
\hline Pack-years & $42.2 \pm 34.8$ & $40.7 \pm 28.2$ & 0.638 \\
\hline \multicolumn{4}{|l|}{ Comorbidities } \\
\hline None & $18(38)$ & $25(58)$ & 0.091 \\
\hline $\mathrm{CCl}$ & $0.52 \pm 0.77$ & $0.67 \pm 0.94$ & 0.777 \\
\hline Body temperature $\left({ }^{\circ} \mathrm{C}\right)^{*}$ & $36.4 \pm 0.5$ & $36.8 \pm 0.6$ & 0.095 \\
\hline \multicolumn{4}{|l|}{ Blood tests } \\
\hline WBC counts $(/ \mu \mathrm{l})$ & $6.736 \pm 2.515$ & $6.214 \pm 2.523$ & 0.186 \\
\hline CRP (mg/dl) & $0.8 \pm 1.4$ & $0.9 \pm 2.3$ & 0.219 \\
\hline
\end{tabular}

'Twenty-six patients (without $A B x(n=17)$ and with $A B x(n=9))$ had the concrete data of temperature at endobronchial ultrasound-guided transbronchial needle aspiration. The temperature of the other patients was below $37.7^{\circ} \mathrm{C}$ at the examination. ABx: Antibiotics; $\mathrm{CCl}$ : Charlson Comorbidity Index; CRP: C-reactive Protein; WBC: White Blood Cell.

Table 1: The baseline characteristics of the enrolled patients. The data are presented as the mean \pm the standard deviation or as the number (percentage), unless otherwise denoted.

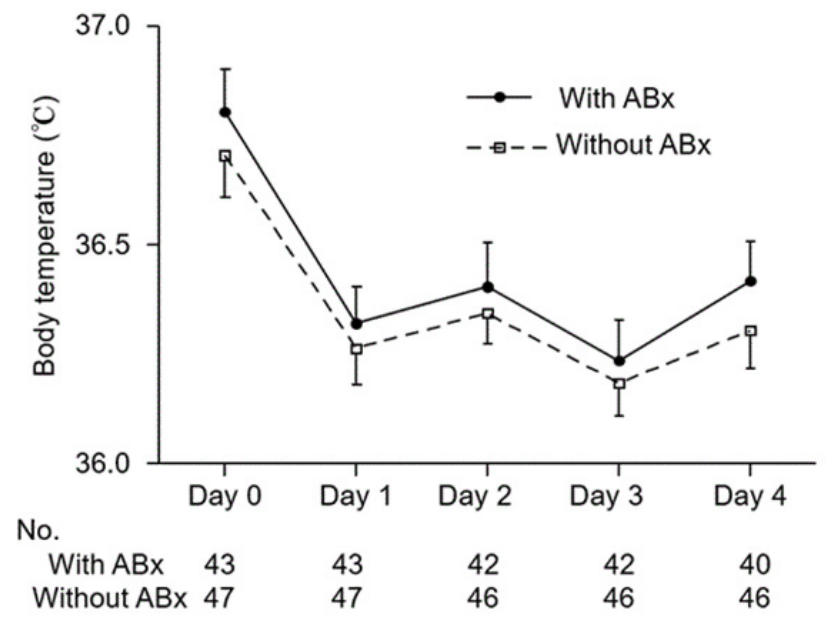

Figure 2: The efficacy of antibiotic prophylaxis within 5 days after endobronchial ultrasound-guided transbronchial needle aspiration. The black circles represent the group that received prophylactic antibiotics $(n=43)$ and the open circles represent the group without antibiotic treatment $(n=47)$. The body temperature was compared between the groups and the data were analyzed using linear mixed models. The data are expressed as the mean \pm the standard deviation. $A B x$ : Antibiotics; CRP: C-reactive Protein; EBUS-TBNA: Endobronchial UltrasoundGuided Transbronchial Needle Aspiration; WBC: White Blood Cells.

EBUS-TBNA; it then gradually decreased. However, the BT did not significantly differ between the groups. A past report has shown that postbronchoscopy fever after fiberoptic bronchoscopy mostly occurred within 12 hours after the procedure [21]. Our result was consistent with previous data.

\section{Other outcome measurements}

The postprocedure WBC and CRP were measured at 10.2 (2 21) days in the control and $11.9(2-24)$ days in the antibiotics group after EBUS-TBNA, respectively. Changes in the WBC and CRP value before and after EBUS-TBNA in the control and antibiotics groups are presented in Figure 3. The baseline and postprocedure WBC and CRP values did not differ between the two groups. The results of the EBUSTBNA procedure are shown in Table 2. In both groups, frequency of postbronchoscopy fever after EBUS-TBNA were 9\%, but EBUS-TBNA-
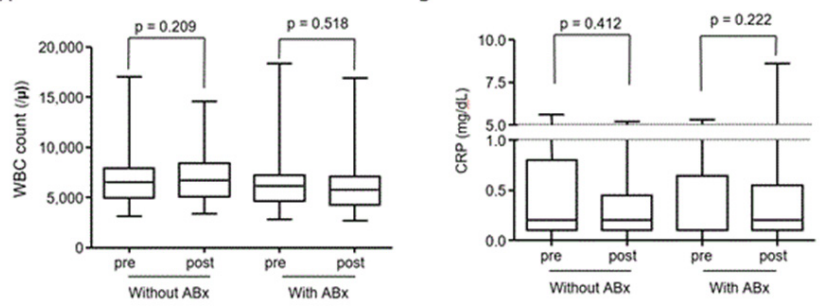

Figure 3: The effect of antibiotic prophylaxis on the white blood count and the C-reactive protein value. Changes in (A) the WBC and (B) the CRP valuespre- and post-EBUS-TBNA in patients with antibiotic prophylaxis $(n=47)$ and without antibiotic prophylaxis $(n=43)$. Changes in the WBC and CRP value were compared by using the Wilcoxon signed-rank test. The data are expressed as the mean \pm the standard deviation. ABx: Antibiotics; data are expressed as the mean \pm the standard deviation. ABX: Antibiotics; Transbronchial Needle Aspiration; WBC: White Blood Cells.

\begin{tabular}{|l|c|c|c|}
\hline & $\begin{array}{l}\text { Without Abx } \\
(\mathbf{n = 4 7 )}\end{array}$ & $\begin{array}{l}\text { With Abx } \\
(\mathbf{n = 4 3 )}\end{array}$ & $\begin{array}{l}\text { p } \\
\text { value }\end{array}$ \\
\hline No. of aspirations & $3.23 \pm 1.96$ & $3.11 \pm 1.12$ & 0.393 \\
\hline Diagnosis & $37(79)$ & $30(70)$ & 0.346 \\
\hline $\begin{array}{l}\text { No. of patients diagnosed by EBUS- } \\
\text { TBNA }\end{array}$ & $26(55)$ & $24(56)$ & 1 \\
\hline Malignant LN metastasis & $92.3 \%(24 / 26)$ & $91.7 \%(22 / 24)$ & 1 \\
\hline Sensitivity & $100 \%(24 / 24)$ & $100 \%(22 / 22)$ & 1 \\
\hline Specificity & $13(28)$ & $12(28)$ & 1 \\
\hline Sarcoidosis & $92.3 \%(12 / 13)$ & $66.7 \%(8 / 12)$ & 0.16 \\
\hline Sensitivity & $100 \%(12 / 12)$ & $100 \%(8 / 8)$ & 1 \\
\hline Specificity & $2(4)$ & 0 & 0.495 \\
\hline Others & \multicolumn{3}{|l|}{} \\
\hline Adverse events & $4(9)$ & $4(9)$ & 1 \\
\hline Fever $\geq 37.8^{\circ} \mathrm{C}$ & 0 & 0 & 1 \\
\hline Infectious complications & - & $6(14)$ & - \\
\hline ABx-related events & - & $5(12)$ & - \\
\hline Diarrhea & - & $1(2)$ & - \\
\hline Rash & \multicolumn{3}{|c|}{0} \\
\hline
\end{tabular}

Table 2: The results of EBUS-TBNA procedure with and without antibiotics. The data is presented as the mean \pm the standard deviation or as the mean (percentage), unless otherwise denoted. ABx: Antibiotics; EBUS-TBNA: Endobronchial Ultrasound-Guided Transbronchial Needle Aspiration; LN: Lymph Node.

associated infectious complications that required antibiotic treatment did not occur within 28 days in either group. The number of punctures did not significantly differ between the groups. The percentage of patients in both groups who were finally diagnosed by EBUS-TBNA was almost similar. The diagnostic sensitivity of malignant LNs metastasis was $92.3 \%$ in the control group and $91.7 \%$ in the antibiotics group. These data were consistent to those of previous reports [22].

Sarcoidosis was diagnosed by compatible pathological findings such as epithelioid granuloma without necrosis in addition to the clinical and radiological characteristics of sarcoidosis. The diagnostic sensitivity for sarcoidosis tended to be higher in the control group (92.3\%) than in the antibiotics group (66.7\%), but this difference was not significant between the groups. Diagnostic specificities of malignant LNs metastasis and sarcoidosis by EBUS-TBNA were 100\% in both groups. Although a grade 1 diarrhea and rash by common terminology criteria for adverse events were observed only in antibiotics group, treatment of prophylactic antibiotics was tolerable (Table 3). 


\begin{tabular}{|l|l|l|r|}
\hline \multicolumn{2}{|l|}{ Without fever (n=82) } & With fever (n=8) & p value \\
\hline Age $(\mathrm{y})$ & $58.9 \pm 14.7$ & $59.8 \pm 13.4$ & 0.989 \\
\hline Male sex & $49(60)$ & $8(100)$ & 0.025 \\
\hline Smoking history & \multicolumn{3}{|r|}{} \\
\hline Never smoker & $12(15)$ & $76.7 \pm 46.4$ & 0.591 \\
\hline Pack-years & $40.3 \pm 27.6$ & 0.011 \\
\hline Comorbidities & $38(46)$ & $5(63)$ & 0.472 \\
\hline None & $1.0 \pm 0.8$ & 0.29 \\
\hline CCI & $0.5 \pm 0.9$ & $4(50)$ & 1 \\
\hline Patients with ABx & $39(48)$ & $6.541 \pm 1.274$ & 0.528 \\
\hline Blood tests & $3.0 \pm 4.5$ & 0.089 \\
\hline WBC count $(/ \mu \mathrm{L})$ & $6.484 \pm 2.624$ & 0.367 \\
\hline CRP (mg/dL) & $0.6 \pm 1.1$ & $3.9 \pm 2.4$ \\
\hline EBUS-TBNA & $3.1 \pm 1.5$ & $5(63)$ & 0.728 \\
\hline No. aspirations & $45(55)$ &
\end{tabular}

Table 3: Comparison of the clinical findings of patients with and without fever The data are presented as the mean \pm the standard deviation or as the mean (percentage), unless otherwise denoted. ABx: Antibiotics; $\mathrm{CCl}$ : Charlson Comorbidity Index; CRP: C-reactive Protein; EBUS-TBNA: Endobronchia Ultrasound-Guided Transbronchial Needle Aspiration; LN: Lymph Node; WBC: White Blood Cell.

\section{Baseline characteristics of patients without fever and with fever}

The baseline characteristics of patients with and without fever were not different with regard to age, sex, presence of comorbidities, and antibiotic prophylaxis. There were also no differences in the WBC and CRP values at baseline, the number of punctures, and the frequency of low-density areas in LNs between the groups, although the smoking pack-year values were significantly higher in the group with fever than in the group without fever.

\section{Discussion}

This preliminary prospective study is the first report to evaluate the efficacy of antibiotic prophylaxis after EBUS-TBNA. The antibiotic treatment had no inhibitory effect on postbronchoscopy fever after EBUS-TBNA. Antibiotic prophylaxis also had no effect on the WBC and CRP value between baseline and postprocedure. The preventive effect of antibiotic prophylaxis on adverse infectious events was unclear because no serious EBUS-TBNA associated infectious complication occurred within 28 days in the groups with and without antibiotics. We were, first, interested in the preventive effect of antibiotic prophylaxis for the serious infectious complications after EBUS-TBNA. However, the frequency of infectious complications following EBUS-TBNA was unclear at the time we started this study, and it was difficult to determine the appropriate sample size for the clinical study. Thus, we focused the fever following EBUS-TBNA as a primary outcome, and planed this preliminary study to detect the efficacy of prophylactic antibiotics after EBUS-TBNA.

Temporary fever after diagnostic fiberoptic bronchoscopy (i.e., postbronchoscopy fever) commonly occurs. Postbronchoscopy fever after the conventional fiberoptic bronchoscopy was found in 10.6$22.6 \%$ of patients in past decade reports [20,21]. Whereas the frequency of postbronchoscopy fever following EBUS-TBNA was still unknown, our study showed firstly that $9.0 \%$ of patients had fever after EBUSTBNA. Previous reports showed that the cause of postbronchoscopy fever may be because of the rise of inflammatory cytokines associated with tissue destruction and/or bacteremia [23]. The elevation of serum levels of inflammatory cytokines such as interleukin (IL)-1 $\beta$, IL-6, and tumor necrosis factor alpha (TNF- $\alpha$ ) have also been reported in patients who developed a fever above $38^{\circ} \mathrm{C}$ after bronchoscopy. There was a highly significant correlation between IL- 6 value and the number of alveolar macrophages in BAL fluid [24]. On the other hand, it have been demonstrated that the frequency of bacteremia were $6.5 \%-8.2 \%$ after conventional fiberoptic bronchoscopy $[20,25,26]$ and $7.0 \%$ after EBUS-TBNA (26), including some febrile patients. Coagulase negative staphylococcus was the most common pathogens and coagulase positive staphylococcus and streptococcus species were also in the blood culture after examinations. These pathogens were often detected in serious infections after EBUS-TBNA [27], and this result may suggest the possibility that bacteremia after EBUS-TBNA may lead to serious infectious complications.

No evidence exists concerning the usefulness of antibiotic prophylaxis after conventional fiberoptic bronchoscopy. A recent randomized study evaluated the effect of prophylactic oral amoxicillin/ clavulanate on postbronchoscopy fever; however, the frequency of fever and the serum levels of pyrogenic cytokines did not differ between the control and antibiotics groups [28]. Another investigator has reported that the frequency of respiratory tract infections after bronchoscopy tended to be lower in the group with prophylactic treatment of azithromycin than in the control group; however, this finding was not significant statistically [19]. Based on these results, the British Thoracic Society Guidelines for diagnostic and therapeutic bronchoscopy does not recommend antibiotic prophylaxis after conventional fiberoptic bronchoscopy, except for patients with specific conditions such as postsplenectomy, postheart valve prosthesis implantation, and previous episode of infectious endocarditis $[29,30]$. Meanwhile, several infectious complications following EBUS-TBNA such as mediastinitis, pneumonia, and pericarditis have been reported [27]. These infectious events were often more serious than those occurring after conventional fiberoptic bronchoscopy, and surgical treatment was required in several cases because systemic antibiotics therapy was insufficient for controlling the infections. Therefore, respiratory physicians are interested in the necessity of antibiotic prophylaxis especially following EBUS-TBNA, and we guess that prophylactic antibiotics may be treated without definite criteria or evidences. One of secondary outcome was preventive effect of serious infectious complications by prophylactic antibiotics in this preliminary study, and those kind of infectious events did not occur. Recent large multicenter-retrospective studies have shown that serious infectious complications after EBUS-TBNA are very rare $(0.16 \%-0.19 \%)[15,31]$. It was unclear whether prophylactic antibiotics had been used to treat patients in past reviews; nevertheless, precautions against such rare complications may be unnecessary.

We also assessed clinical characteristics of patients with and without fever after EBUS-TBNA. Our results showed that existing of comorbidities and number of punctures were not risk factor of postbronchoscopy fever, and antibiotic treatment was also unrelated to the development of fever following EBUS-TBNA. Smoking packyears was significantly higher in the group with fever than in the group without fever. Patients with a longer smoking history are included in the high-risk group with respiratory tract infection. We suspected febrile patients with smoking history after EBUS-TBNA might have acute episode of bronchitis, but serious infectious events did not occur even in those patients. Low-density areas in LNs, as detected by computed tomography scanning, generally indicate tissue necrosis. However, the puncture of LNs with low-density areas did not elevate the frequency of postbronchoscopy fever in this study. The risk of infectious complications after puncturing a cystic lesion was demonstrated in a past report [31]. Patient selection, thus, could be more important in preventing infectious adverse events. In addition, cleaning the oral 
Citation: Takagi H, Nagaoka T, Ando K, Tsutsumi T, Ichikawa M, et al. (2017) Efficacy of Antibiotic Prophylaxis after Endobronchial Ultrasound-guided Transbronchial Needle Aspiration: A Preliminary Prospective Study. J Pulm Respir Med 7: 416. doi: 10.4172/2161-105X.1000416

Page 5 of 5

mucosa and the aspiration needle before the puncture may be useful in reducing infectious complications associated with EBUS-TBNA.

There were several limitations in this study. First, preventive effect of serious infectious complications by antibiotics prophylaxis was still unclear, because the sample size of this preliminary study was too small for evaluation. Second, exclusion of over $20 \%$ of enrolled participants because of protocol violation decreased the quality of this study. Third, there were only two participating institutions, and the examination procedure may have been biased. Forth, the values of WBC and CRP may have been biased, because the time measuring blood test after EBUS-TBNA was not unified. Fifth, we did not evaluate bacteremia and inflammatory cytokines in any patient; thus, the causes of fever were unclear for each febrile patient.

\section{Conclusion}

The findings of this preliminary study and the low frequency of infectious complications, which has also been demonstrated in previous reports, suggest that antibiotic prophylaxis is not recommended for EBUS-TBNA as same with the guideline for conventional diagnostic fiberoptic bronchoscopy. Further investigation of the phenotype of patients with infectious complications after EBUS-TBNA may be useful to detect patients who need selective treatment by prophylactic antibiotics.

\section{References}

1. Vaidya PJ, Kate AH, Yasufuku K, Chhajed PN (2015) Endobronchial ultrasoundguided transbronchial needle aspiration in lung cancer diagnosis and staging. Expert Rev Respir Med 9: 45-53.

2. Murgu SD (2015) Diagnosing and staging lung cancer involving the mediastinum. Chest 147: 1401-1412.

3. Kinsey CM, Arenberg DA (2014) Endobronchial ultrasound-guided transbronchial needle aspiration for non-small cell lung cancer staging. Am J Respir Crit Care Med 189: 640-649.

4. Agarwal R, Srinivasan A, Aggarwal AD, Gupta D (2012) Efficacy and safety of convex probe EBUS-TBNA in sarcoidosis: a systematic review and metaanalysis. Respir Med 106: 883-892.

5. Grosu HB, Iliesiu M, Caraway NP, Medeiros LJ, Lei X, et al. (2015) Endobronchial ultrasound guided transbronchial needle aspiration accurately diagnoses and subtypes lymphoma. Ann Am Thorac Soc 12: 1336-1344.

6. Kennedy MP, Jimenez CA, Bruzzi JF, Mhatre AD, Lei X, et al. (2008) Endobronchial ultrasound-guided transbronchial needle aspiration in the diagnosis of lymphoma. Thorax 63: 360-365.

7. Yang B, Li F, Shi W, Liu H, Sun S, et al. (2014) Endobronchial ultrasoundguided transbronchial needle biopsy for the diagnosis of intrathoracic lymph node metastases from extrathoracic malignancies: a meta-analysis and systematic review. Respirology 19: 834-841.

8. Yasufuku K, Nakajima T, Fujiwara T, Yoshino I, Keshavjee S (2011) Utility of endobronchial ultrasound-guided transbronchial needle aspiration in the diagnosis of mediastinal masses of unknown etiology. Ann Thorac Surg 91: 831-836.

9. Yasufuku K, Pierre A, Darling G, de Perrot M, Waddell T, et al. (2011) A prospective controlled trial of endobronchial ultrasound-guided transbronchial needle aspiration compared with mediastinoscopy for mediastinal lymph node staging of lung cancer. J Thorac Cardiovasc Surg 142: 1393-1400.

10. Shingyoji M, Nakajima T, Yoshino M, Yoshida Y, Ashinuma H, et al. (2014) Endobronchial ultrasonography for positron emission tomography and computed tomography-negative lymph node staging in non-small cell lung cancer. Ann Thorac Surg 98: 1762-1767.

11. Nakajima T, Yasufuku K, Nakagawara A, Kimura H, Yoshino I (2011) Multigene mutation analysis of metastatic lymph nodes in non-small cell lung cancer diagnosed by endobronchial ultrasound-guided transbronchial needle aspiration. Chest 140: 1319-1324.

12. Sakairi Y, Nakajima T, Yasufuku K, Ikebe D, Kageyama H, et al. (2010) EML4ALK fusion gene assessment using metastatic lymph node samples obtained by endobronchial ultrasound-guided transbronchial needle aspiration. Clin Cancer Res 16: 4938-4945.

13. Neat MJ1, Foot NJ, Hicks A, Breen R, Wilkins B (2013) ALK rearrangements in EBUS-derived transbronchial needle aspiration cytology in lung cancer. Cytopathology 24: 356-364

14. Varela-Lema L, Fernández-Villar A, Ruano-Ravina A (2009) Effectiveness and safety of endobronchial ultrasound-transbronchial needle aspiration: A systematic review. Eur Respir J 33: 1156-1164.

15. Asano F, Aoe M, Ohsaki Y, Okada Y, Sasada S, et al.(2013) Complications associated with endobronchial ultrasound-guided transbronchial needle aspiration: A nationwide survey by the Japan Society for Respiratory Endoscopy. Respir Res 14:50.

16. Charlson ME, Pompei P, Ales KL, MacKenzie CR (1987) A new method of classifying prognostic comorbidity in longitudinal studies: development and validation. J Chronic Dis 40: 373-383.

17. Nakajima T, Yasufuku K (2011) How I do it-Optimal methodology for multidirectional analysis of endobronchial ultrasound-guided transbronchial needle aspiration samples. J Thorac Oncol 6: 203-206.

18. Dan L, Anthony F, Dennis K, Stephen H, Jameson J, et al. (2011) Harrison's Principles of Internal Medicine (18 ${ }^{\text {th }}$ Edtn). McGraw-Hill Professional, New York, US.

19. Kanazawa H (2007) Efficacy of azithromycin administration in prevention of respiratory tract infection after bronchoscopic biopsy: A randomized, controlled trial. Respirology 12: 70-75.

20. Sharif-Kashani B, Shahabi P, Behzadnia N, Mohammad-Taheri Z, Mansour $D$, et al. (2010) Incidence of fever and bacteriemia following flexible fiberoptic bronchoscopy: A prospective study. Acta Med Iran 48: 385-388.

21. Park JS, Lee CH, Yim JJ, Yang SC, Yoo CG, et al. (2011) Impact of antibiotic prophylaxis on postbronchoscopy fever: A randomised controlled study. Int J Tuberc Lung Dis 15: 528-535.

22. Medford AR, Bennett JA, Free CM, Agrawal S (2010) Endobronchial ultrasoundguided transbronchial needle aspiration (EBUS-TBNA): Applications in chest disease. Respirology 15: 71-79.

23. Picard E, Schwartz S, Goldberg S, Glick T, Villa Y, et al. (2000) A prospective study of fever and bacteremia after flexible fiberoptic bronchoscopy in children. Chest 117: 573-577.

24. Krause A, Hohberg B, Heine F, John M, Burmester GR, et al. (1997) Cytokines derived from alveolar macrophages induce fever after bronchoscopy and bronchoalveolar lavage. Am J Respir Crit Care Med 155: 1793-1797.

25. Yigla M, Oren I, Bentur L, Solomonov A, Elias N, et al. (1999) Incidence of bacteraemia following fibreoptic bronchoscopy. Eur Respir J 14: 789-791.

26. Steinfort DP, Johnson DF, Irving LB (2010) Incidence of bacteraemia following endobronchial ultrasound-guided transbronchial needle aspiration. Eur Respir J 36: 28-32.

27. Ishimoto $\mathrm{H}$, Yatera $\mathrm{K}$, Uchimura $\mathrm{K}$, Oda $\mathrm{K}$, Takenaka M et al. (2015) A serious mediastinum abscess induced by endobronchial ultrasound-guided transbronchial needle aspiration (EBUS-TBNA): A case report and review of the literature. Intern Med 54: 2647-2650.

28. Yamamoto M, Nagano T, Okuno K, Nakata K, Takenaka K, et al. (2012) An open-label, prospective clinical study to evaluate the efficacy of prophylactic antibiotics after diagnostic bronchoscopy. Kobe J Med Sci 58: E110-E118.

29. Du Rand IA, Blaikley J, Booton R, Chaudhuri N, Gupta V, et al. (2013) Summary of the British Thoracic Society guideline for diagnostic flexible bronchoscopy in adults. Thorax 68: 786-787.

30. Du Rand IA, Barber PV, Goldring J, Lewis RA, Mandal S, et al. (2011) Summary of the British thoracic society guidelines for advanced diagnostic and therapeutic flexible bronchoscopy in adults. Thorax 66:1014-1015.

31. Çaglayan B, Yilmaz A, Bilaçeroglu S, Comert SS, Demirci NY, et al. (2016) Complications of convex-probe endobronchial ultrasound-guided transbronchial needle aspiration: A multi-center retrospective study. Respir Care 61: 243-248. 